\title{
Improving the Utilization Rate of Insulin Pump in Patients with Diabetes Mellitus with Toyota TBP
}

\author{
Xixi Luo ${ }^{\dagger}$, Yan Zhou ${ }^{\dagger}$, Xiaohua Lu*, Wane Zhao, Yan Liu, Qinling Chen \\ Department of Endocrinology, The First Affiliated Hospital of Jinan University, Guangzhou, China
}

Email address:

536414169@qq.foxmail.com (Xiaohua Lu)

${ }^{*}$ Corresponding author

$\dagger$ Xixi Luo and Yan Zhou are co-first authors.

\section{To cite this article:}

Xixi Luo, Yan Zhou, Xiaohua Lu, Wane Zhao, Yan Liu, Qinling Chen. Improving the Utilization Rate of Insulin Pump in Patients with Diabetes Mellitus with Toyota TBP. American Journal of Nursing Science. Vol. 10, No. 2, 2021, pp. 133-137. doi: 10.11648/j.ajns.20211002.14

Received: February 13, 2021; Accepted: March 13, 2021; Published: March 30, 2021

\begin{abstract}
Objective: To examine the efficacy of Toyota Business Practice (TBP) method in improving the utilization rate of insulin pump in non-endocrine diabetic patients. Methods: Toyota method is a process requires continuous learning and improvement. The characteristic feature of Toyota's working method is the problem-solving guided by basic consciousness. It is the essential basic knowledge for every person who studies Toyota problem solving methods. TBP is an ideal and scientific tool for achieving goals, careful planning, effective implementation, timely review and effective measures are the ways to make TBP a more efficient and economical tool. It is divided into eight steps including clarify problems, decompose problems, set goals, identify the real causes, formulate countermeasures, implement, evaluate results and processes, as well as consolidate achievements were used in the process of prescribing insulin pump to patients with diabetic treated in non-endocrine department. The efficacy of Toyota TBP will be measured and compared in a cohort before and after the implantation. Results: After utilizing Toyota TBP method, the use of insulin pump in non-endocrinology department increased by $10 \%$. Conclusion: Our data suggested that the Toyota TBP method optimized the process of using insulin pump in non-endocrinology department, improved the work flow and the overall utilization rate as well as the prognosis of patients.
\end{abstract}

Keywords: Toyota TBP Method, Non Endocrinology Department, Diabetic Patients, Insulin Pump Utilization Rate

\section{Introduction}

According to the most recent data of the International Diabetes Federation (IDF) published in 2017 [1], there were 425 million adult patients with diabetes in the world which would reach 629 million by 2045 . Over the same period, China had about 1.144 billion adult patients with diabetes mellitus in the mainland which accounting for $25 \%$ of the total number of diabetic patients worldwide. With the aging of population and changing of diet, the number of diabetic patients in China has increased rapidly in last 30 years. At present, many patients with diabetes are receiving treatment in non-endocrinology department. Insulin pump uses artificial intelligence controlled insulin input device to mimic the physiological secretion of insulin to the greatest extent through continuous subcutaneous infusion of insulin to achieve better control of blood glucose [2]. With the increasing popularity of insulin pump among diabetic patients, the occurrence of diabetes associated organ damage has been reduced. TBP is a modern management system. By exploring the potential creativity and management ability of on-site staff, it can cultivate the ability of all staff to find and solve problems spontaneously. In recent years, it has been applied in the field of medicine to reduce the waste of time and resource [3-5] However, insulin pump is rarely prescribed in non-endocrinology department. In 2016, the Department of endocrinology of our hospital officially launched the project named Blood Glucose Management. Ever since, the insulin pump has been used in non-endocrinology department under the recommendations form endocrinologists. From 2016 to 2019, the utilization rate of insulin pump in non-endocrinology department showed an overall upward trend. However, the utilization rate of insulin pump in non-endocrinology department was still relatively low compared to endocrinology department. Therefore, in this study, we applied Toyota TBP method to improve the utilization rate of insulin pump and evaluated its efficacy in a cohort. 


\section{Data and Methods}

\subsection{Study Population}

The data of all patients who used insulin pumps in non-endocrinology Department of our hospital from January 2016 to August 2020 were retrospectively collected and analyzed.

\subsection{Toyota TBP}

The processes of Toyota TBP [6-7] were defined as: clarify problems, decompose problems, set goals, identify the real causes, formulate countermeasures, implement, evaluate results and processes, as well as consolidate achievements.

\subsubsection{Clarify Problems}

The data suggested that the only 99 patients used insulin pumps from May to August in 2020, which was far less than the 152 cases from May to August in 2019 (see table 1 and table 2).

Table 1. Use of insulin pump in non-endocrinology Departments from May to August from 2016 to 2020 (case).

\begin{tabular}{|c|c|c|c|c|c|c|}
\hline Month/year & $2016 Y$ & $2017 Y$ & $2018 Y$ & 2019Y & $2020 Y$ & Proportion of the same period between 2020 and 2019 \\
\hline May & 2 & 2 & 39 & 36 & 15 & $42.0 \%$ \\
\hline June & 1 & 5 & 40 & 40 & 25 & $63.0 \%$ \\
\hline July & 0 & 1 & 41 & 35 & 29 & $83.0 \%$ \\
\hline Total & 3 & 13 & 150 & 152 & 99 & $65.0 \%$ \\
\hline
\end{tabular}

Table 2. Prediction and comparison of the top 5 departments in the use of insulin pump outside the Endocrinology Department from May to August in 2019.

\begin{tabular}{|c|c|c|c|c|c|}
\hline & $\begin{array}{l}\text { pump cases in } \\
2019\end{array}$ & $\begin{array}{l}\text { aim pump } \\
\text { cases in } 2020\end{array}$ & $\begin{array}{l}\text { Forecast for pump } \\
\text { cases in } 2020\end{array}$ & $\begin{array}{l}\text { actual pump cases } \\
\text { in } 2020\end{array}$ & $\begin{array}{l}\text { Proportion of the same period } \\
\text { between } 2020 \text { and } 2019\end{array}$ \\
\hline 1. Orthopedics West & 28 & 30 & 35 & 8 & $28.6 \%$ \\
\hline 2. Orthopedics East & 14 & 15 & 20 & 8 & $57.1 \%$ \\
\hline 3. Gastroenterology & 13 & 14 & 16 & 5 & $38.5 \%$ \\
\hline 4. Neurology & 12 & 13 & 15 & 8 & $66.7 \%$ \\
\hline 5. Plastic surgery & 9 & 10 & 16 & 6 & $66.7 \%$ \\
\hline
\end{tabular}

\subsubsection{Establishment of a Blood Glucose Management Quality Improvement Team}

In order to achieve the ultimate goal of "improving the utilization rate of insulin pump in patients from non-endocrinology Departments", the Toyota TBP method was applied by the blood glucose management team. After analyzing the data, the biggest problem was identified as the $35 \%$ difference from expected use of insulin pump to actual use. To better identified the root causes, a quality improvement team (including diabetes specialist nurses, blood glucose management doctors and the key person of the department) was established.

\subsubsection{Decomposition of Problem}

i. The team first conducted a questionnaire survey with patients treated by insulin pump from non-endocrinology departments. It appeared that about $10 \%$ of all patients worried about blood glucose management during the insulin pump treatment, and more than $90 \%$ of the patients appreciated the effect of insulin pump and willing to continue to be treated with insulin pump treatment. On the other hand, $12.5 \%$ of the medical staff concerned about the blood glucose management during the insulin pump treatment, and more than $90 \%$ of the medical staff appreciated the effect of insulin pump and willing to prescribe. (see Table 3 and table 4).

Table 3. A survey of factors related to non endocrine patients.

\begin{tabular}{lllll}
\hline & $\begin{array}{l}\text { Worry about blood glucose management } \\
\text { during insulin pump treatment }\end{array}$ & $\begin{array}{l}\text { Inconvenience of } \\
\text { insulin pump }\end{array}$ & $\begin{array}{l}\text { Obvious efficacy of } \\
\text { insulin pump therapy }\end{array}$ & $\begin{array}{l}\text { Satisfied with insulin } \\
\text { pump therapy }\end{array}$ \\
\hline Proportion of patients & $10 \%$ & $10 \%$ & $90 \%$ & $100 \%$ \\
\hline
\end{tabular}

Table 4. A survey of factors related to in non endocrine medical staff.

\begin{tabular}{llllll}
\hline & $\begin{array}{l}\text { Worry about } \\
\text { blood glucose } \\
\text { management }\end{array}$ & $\begin{array}{l}\text { Consider insulin } \\
\text { subcutaneous injection as } \\
\text { the first choice }\end{array}$ & $\begin{array}{l}\text { Worry } \\
\text { about Pump } \\
\text { cost }\end{array}$ & $\begin{array}{l}\text { Proper } \\
\text { pump speed }\end{array}$ & $\begin{array}{l}\text { considered that the } \\
\text { efficacy of pump } \\
\text { therapy was obvious }\end{array}$ \\
\hline 1. Proportion of patients & $12.5 \%$ & $68.8 \%$ & $25 \%$ & $100 \%$ & $100 \%$ \\
2. Proportion of patients & $12.8 \%$ & $58.8 \%$ & $11.8 \%$ & $100 \%$ & $90 \%$ \\
\hline
\end{tabular}

ii. The process of using insulin pump outside Endocrinology department

According to the protocol, four steps were required for the use of insulin pump outside the endocrinology department: (1) applying insulin pump therapy (2) daily insulin pump Patrol (3) stop insulin pump therapy (4) feedback and follow-up of insulin pump of regulating blood glucose. Through the investigation, we found that most problem lied in the first section which emphasized on the timing and method of applying insulin pump therapy.

iii. Identification of the reasons for not using insulin pump treatment.

Two hypotheses had been proposed for the low application rate of insulin pump. Firstly, in the first half of this year, affected 
by the new coronavirus, the number of patients in the hospital was decreased. As a matter of fact, the use of insulin pump in non endocrinology department was suspended due to the epidemic situation from January to April this year. Secondly, most doctors still consider subcutaneous injection of insulin as the first choice unless the blood glucose was very difficult to control.

\subsubsection{Identify the Real Causes}

The most common reason for not prescribing insulin pump was the policy change during the pandemic in the first of the year. Another common reply was that the doctor would not consider using insulin pump unless the blood glucose more than 16.8 $\mathrm{mmol} / \mathrm{L}$. Additionally, some other doctors complained about the inconvenience about prescribing insulin pump such as the pump can be installed only in the morning. In the first half of the year, the insulin pump service was stopped for 4 months in non -endocrinology departments. However, the relevant departments were not properly informed after the service restarted. Our survey suggested that the advantages of insulin pump and the standard process of insulin pump installation should be better informed to doctors and nurses outside the endocrinology department.

\subsubsection{Set Goals}

It is necessary to minimize the loss of stopping external pump treatment due to the influence of new coronavirus at the beginning of this year which strive to increase the number of pumps from September to December in the second half of the year by $10 \%$ or more on the basis of the same period last year.

\subsubsection{Formulate Countermeasures and Implementation Plan}

Support from Endocrinology departments
Next, we communicated with doctors from other department and adjust the previous protocol of setting pump treatment and prolonged the working time to whole day instead of morning only.

We should strengthen the training and promotion of the advantages and standard procedure of insulin pump for non-endocrinology doctors and nurses and distribute the self-made medical manual of insulin pump for blood glucose management to all departments as well as communicate with the director and head nurse of relevant departments. According to the contents of the medical manual, restart the training of insulin pump including 8 key departments (Orthopedics, Gastrointestinal etc.) but the team maintained wechat communication with doctors in other departments as well. Upon request, doctors on duty of the endocrinology depart was requested to provide necessary consultation and help in time.

We also encouraged frequent check of the use of insulin pump in non-endocrinology departments with proper suggestions if needed. The number of insulin pumps used outside the department and related records were registered by blood glucose management nurses. In order to understand the situation of blood glucose control in patients with insulin pump treatment in different departments; nurses and insulin pump specialist from blood glucose management team checked the implementation rates every week and recorded the problems in the implementation process before improvements and changes could be made to further smooth the process [6-7]. We had generated a flow chart depict the process of using non endocrine insulin pump (as shown in the figure below).

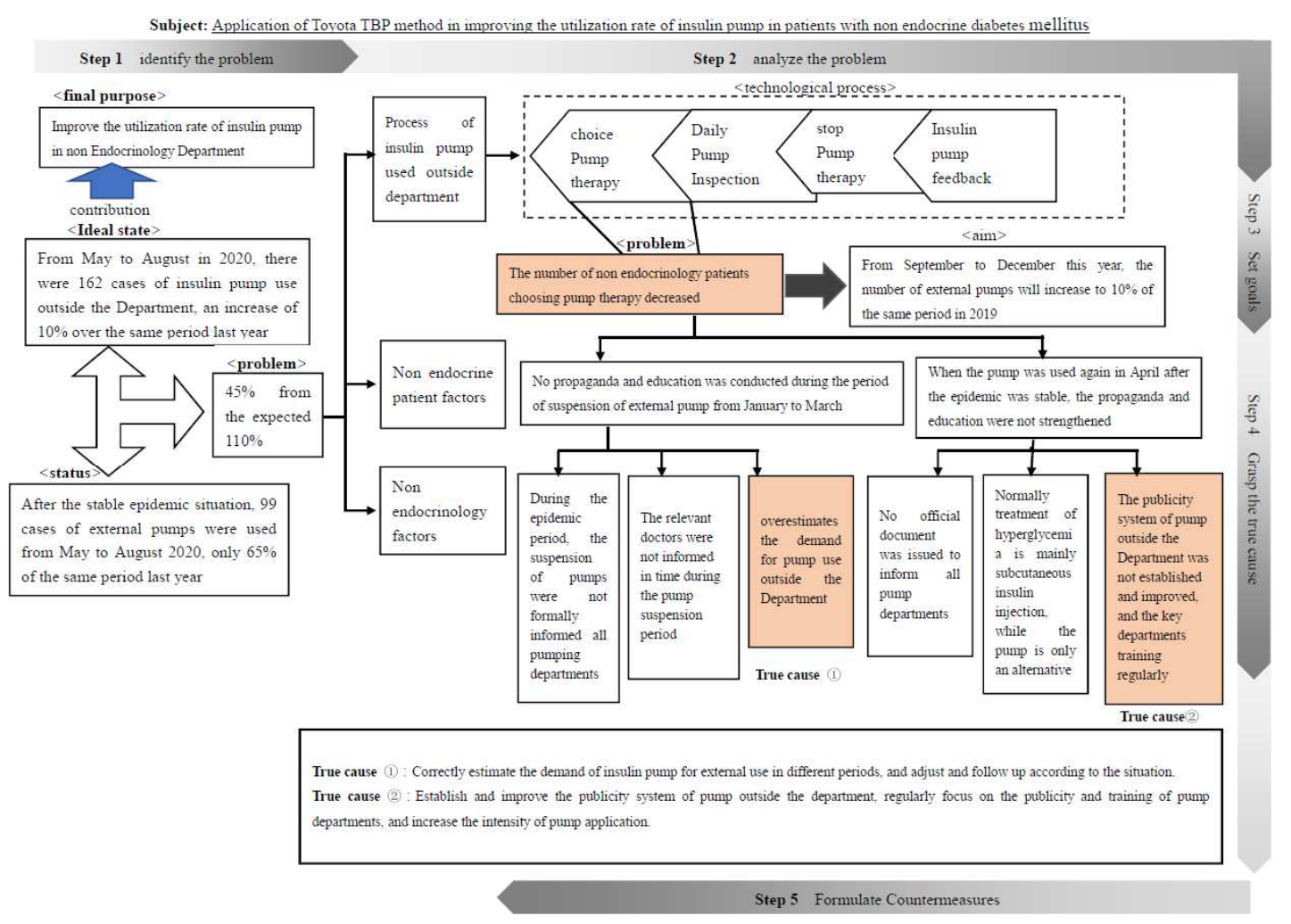

Figure 1. Application of Toyota TBP method in improving the utilization rate of insulin pump in patients with non endocrine diabetes mellitus. 


\subsubsection{Statistical Analysis}

SPSS16.0 software was used for statistical analysis.

\section{Result}

Table 5. Comparison of the use of insulin pump before and after improvement.

\begin{tabular}{lllll}
\hline Item (May to November) & May to August & $\begin{array}{l}\text { Difference between the same } \\
\text { period of 2020 and 2019* }\end{array}$ & September to November & $\begin{array}{l}\text { Difference between the same } \\
\text { period of 2020 and 2019* }\end{array}$ \\
\hline $\begin{array}{l}\text { Number of insulin pumps (case) } \\
\text { 2019Y 276 }\end{array}$ & 152 & 124 & \\
2020Y 235 & 99 (Before improvement) & $65.1 \%$ & 136 (After improvement) & $110 \%$ \\
$\begin{array}{l}\text { Day with pump (day) } \\
\text { 2019Y 2465 }\end{array}$ & 1295 & & 1170 \\
2020Y 1933 & 820 (Before improvement) & $63.3 \%$ & 1113 (After improvement) & $95.1 \%$ \\
\hline
\end{tabular}

*Proportion of the same period in 2020 and 2019: the proportion obtained by dividing the number of cases or days in the same period in 2020 by the number of cases or days in the same period in 2019

Table 6. Qualified blood glucose and incidence of hypoglycemia in the same period of September to December 2019 and 2020.

\begin{tabular}{lllll}
\hline Item & $\mathbf{2 0 1 9}$ & Proportion of the same period & $\mathbf{2 0 2 0}$ & Proportion of the same period \\
\hline Non operative & & & & $93.1 \%$ \\
Within range & 40 & $87 \%$ & 27 & $6.9 \%$ \\
Outside normal range & 6 & $13 \%$ & 5 & $17.2 \%$ \\
hypoglycemia & 8 & $17.4 \%$ & 24 & $82.8 \%$ \\
No hypoglycemia & 38 & $82.6 \%$ & 39 & $97.5 \%$ \\
Perioperative & & & 1 & $2.5 \%$ \\
Within range & 37 & $94.9 \%$ & 13 & $32.5 \%$ \\
Outside normal range & 2 & $5.1 \%$ & 27 & $67.5 \%$ \\
hypoglycemia & 12 & $30.8 \%$ & & \\
No hypoglycemia & 27 & $69.2 \%$ & & \\
\hline
\end{tabular}

Note: non operative patients did not include pregnant patients and critically ill patients.

\section{Discussion and Conclusion}

Insulin pump can mimic the physiological secretion of insulin to the greatest extent and reduces the variation of insulin absorption as well as reduces the risk of hypoglycemia. More importantly, it has been shown to be associated with less weight gain, better perioperative blood glucose control and compliance of patients [2]. As a continuous subcutaneous insulin infusion device, insulin pump is suitable for all diabetic patients who need insulin treatment. Therefore, the short-term use of insulin pump in non-endocrinology department has been proved to be beneficial to patients [8] Since 2016, our endocrine team has been using insulin pumps to help diabetes patients outside endocrinology department [9]. As suggested in table 6, from September to December of 2020, compared with the same period in 2019, the normalization of glucose among non-surgical patients increased from $87 \%$ to $93.1 \%$. There was no difference in the incidence of hypoglycemia. Among perioperative patients, the normalization of glucose increased from $94.5 \%$ to $97.5 \%$. There was no significant difference in the incidence of hypoglycemia.

Toyota mode of production is a long history of Toyota. It was founded in the era of Toyoda Sasaki. It has been promoted and inherited by Mr. Yoshiro Toyoda until now. The application of Toyota production mode makes Toyota company obtain great benefit and present strong competitiveness [7]. Toyota mode of production is a method of production management which has gradually become the corporate culture of Toyota company. Toyota mode of production can enhance the vitality of the enterprise which is a complete set of production system and methods. Its main purpose is to eliminate waste to the greatest extent, through the rationalization of production to achieve the superiority of products, reasonable manufacturing products which can create the customer concept of quality first. Finally, it can solve problems, improve quality and efficiency.

Toyota's Business Practice (TBP) refers to the specific practice of Toyota's work method in daily work. TBP is mainly implemented through eight specific steps that includes identifying the problem, decomposing the problem, setting goals, understanding the cause, formulating countermeasures, implementing, evaluating the results and processes, as well as consolidating the results. Due to the Covid-19 epidemic in 2020, the utilization rates of insulin pump in non-endocrinology department of our hospital were significantly decreased compared to the same period in 2019. In September, we applied Toyota's work method to analyze and improved the situation. As presented in Table 1, the utilization of insulin pump during May to August in 2020 was only 99 cases, which was far behind the 152 cases during the same period in 2019. After analyzing the data, problems were identified in the process. Further decomposing of the problem suggested that the biggest reason was the low awareness of the doctors in non-endocrinology department for the use of insulin pump and the right timing of using the pump. The second real 
cause was that the pump installation operation system in non-endocrinology department has not been established and improved. According to Toyota's work method process, we optimized the service process, refined the work flow, and increased insulin pump training. After the changes, the number of insulin pumps installation increased by $10 \%$ from September to December in 2020, indicating that Toyota's work method could improve the utilization rate of insulin pumps among non-endocrinology department.

In summary, Toyota method is a process that requires continuous learning and improvement [10-12]. During the process of applying Toyota production mode, we should also pay attention to the improvement and development combined with the actual situation of medical care. Our data suggest that Toyota TBP can improve the efficiency of medical care and save costs. The blood glucose management team used Toyota's work method to analyze the current situation and identified the root causes before successfully formulated countermeasures, that finally improved the utilization rate of insulin pump among non-endocrinology departments. Our study was similar to that of Deng Wanping' study [13-15], which introduced the importance of patient-centered, high-quality and safe medical and nursing services that ensured the smooth implementation of insulin pump in non-endocrinology department. However, larger studies involving more patients and longer follow-up time are needed to prove our statements.

\section{Ethics Approval and Consent to Participate}

Not applicable.

\section{Competing Interests}

The authors declare that they have no competing interests.

\section{Fund}

The work was supported by grants from Guangdong Medical Science and Technology Research Fundation in 2019 (No. B2019012) and Guangdong Medical Science and Technology Research Fundationt in 2020 (No. A2020574).

\section{References}

[1] You liming. Medical Nursing [M]. BeiJing: People's Health Publishing House, 2006: 413.
[2] Endocrinology and metabolism branch of Chinese Medical Association, endocrinology branch of Chinese Medical Association, diabetes branch of Chinese Medical Association, diabetes branch of Chinese Medical Association, Chinese insulin pump treatment guidelines (2014 Edition) $[\mathrm{J}]$ clinical treatment of diabetes, 2014, (08): 353-359.

[3] Wei Lianyan, Xv Dongjuan and etc. Application of quality control circle and Toyota production mode to shorten the time of intravenous thrombolysis in patients with acute cerebral infarction [J]. Modern Chinese doctors, 2019, 57 (15): 4-8.

[4] Li Ran. Inspiration of just in time and automation of Toyota production mode to Chinese manufacturing enterprises [J]. Contemporary tourism, 2018 (07): 119.

[5] Liu Chongyang. Research on the problems and solutions of Toyota production mode in Chinese manufacturing enterprises [J]. China's high tech Enterprises, 2017, (06): 49-50.

[6] Wang Xuejiao. The application of Toyota production mode (TPS) in Fengyue company [D]. Jilin University, 2017.

[7] Mao Gangxin. Application of Toyota production mode in improving engine die [D]. Jilin University, 2020.

[8] AACE Insulin Pump Management Task Force. Statement by the American Association of Clinical Endocrinologists Consensus Panel on insulin pump management [J]. Endocr Pract, 2010, 16 (5): 746-762.

[9] Guo Xiaohui. Chinese insulin pump treatment nursing management standard $[\mathrm{M}]$. Wu Han: Hubei Science and Technology Press, 2018: 1.

[10] Kazuo Wada. The Evolution of the Toyota Production System [M]. Springer, Singapore, 2020.

[11] Treasa 'Susie' Leming-Lee and Shea Polancich and Bonnie Pilon. The Application of the Toyota Production System LEAN 5S Methodology in the Operating Room Setting [J]. Nursing Clinics of North America, 2019, 54 (1): 53-79.

[12] Nicholas Loyd. Content validation of an assessment tool to measure lean deployment based on the Toyota Production System and Toyota Way [J]. Int. J. of Lean Enterprise Research, 2018, 2 (3): 258-285.

[13] Deng Wanping, Chen bing and etc. Continuous quality improvement based on the cause analysis of hypoglycemia nursing in hospitalized diabetic patients (Application of safety management) [J]. Electronic Journal of practical clinical nursing, 2019, 4 (38): 175.

[14] Papa Frank Joseph, Alexander Jerry H. Aggregated student confidence estimates support continuous quality improvements in a competencies-oriented curriculum. [J]. BMJ open quality, 2019, 8 (1).

[15] Lum Brandon, Png Hong Muay, Yap Hock Lan, Tan Cindy, Sun Bixian, Law Yen Hoon. Streamlining workflows and redesigning job roles in the theatre sterile surgical unit. [J]. BMJ open quality, 2019, 8 (3). 